\title{
NOTE ON CUBATURE FORMULAE AND DESIGNS OBTAINED FROM GROUP ORBITS
}

\author{
HIROSHI NOZAKI AND MASANORI SAWA
}

\begin{abstract}
In 1960, Sobolev proved that for a finite reflection group $G$, a $G$-invariant cubature formula is of degree $t$ if and only if it is exact for all $G$-invariant polynomials of degree at most $t$. In this paper, we find some observations on invariant cubature formulas and Euclidean designs in connection with the Sobolev theorem. First, we give an alternative proof of theorems by $\mathrm{Xu}(1998)$ on necessary and sufficient conditions for the existence of cubature formulas with some strong symmetry. The new proof is shorter and simpler compared to the original one by $\mathrm{Xu}$, and moreover gives a general interpretation of the analytically-written conditions of Xu's theorems. Second, we extend a theorem by Neumaier and Seidel (1988) on Euclidean designs to invariant Euclidean designs, and thereby classify tight Euclidean designs obtained from unions of the orbits of the corner vectors. This result generalizes a theorem of Bajnok (2007) which classifies tight Euclidean designs invariant under the Weyl group of type $B$ to other finite reflection groups.
\end{abstract}

\section{INTRODUCTION}

A main problem of numerical integration is to approximate the integral

$$
\int_{\Omega} f(x) d \mu \text {. }
$$

Here $x$ is an $n$-dimensional coordinate vector and $\mu$ is a probability measure on a domain $\Omega$ in $\mathbb{R}^{n}$. We search for an approximation formula by taking a positive linear combination of the function values of $f$ at specified points $x_{1}, \cdots, x_{N}$, that is,

$$
\sum_{i=1}^{N} w_{i} f\left(x_{i}\right) .
$$

We call (1.1) a cubature formula. The values $w_{i}$ are the weights and $x_{i}$ are the points of a cubature formula. To each formula we assign the set of functions for which it is exact. Most often this set is the space of all polynomials of degree no more than $t$; in this case a cubature formula is said to be of degree $t$. We refer the readers to the comprehensive monograph [12, 26] for the basic theory of cubature formula.

A fundamental objective is to construct cubature formulas of large degrees with few points. The requirement that a given cubature formula is exact for polynomials

1991 Mathematics Subject Classification. Primary 65D32, Secondary 05E99, 51M99.

Key words and phrases. Cubature formula, Euclidean design, radially symmetric integral, reflection group, Sobolev theorem.

This research started while the authors were visiting scholars at the University of Texas at Brownsville. This research is supported by the Japan Society for the Promotion of Science. 
up to a certain degree can be reduced to the problem of solving a system of algebraic equations. In general, the larger the number of points or the degree of a cubature formula is, the greater the size of this system is. Sobolev [25] gave a celebrated criterion to diminish the size of the system to be solved. Namely, he proved that an invariant cubature formula is of degree $t$ if and only if it is exact for all polynomials of degree at most $t$ invariant under the group. This is known as the Sobolev theorem. The Sobolev theorem is widely accepted by the cubature community in analysis and related areas; for instance see [19, 24].

Independent of the line of research in analysis and related areas, Goethals and Seidel [14, Theorem 3.12] developed the invariant theory of Chebyshev-type cubature formulas on the sphere or spherical designs. As a generalization of spherical designs Neumaier and Seidel [20] considered cubature formulas on several concentric spheres called Euclidean designs. Bajnok 2] classified tight Euclidean designs whose points are the union of the orbits of the corner vectors of the group $B_{n}$, and in particular, he obtained several new tight designs. Here a Euclidean design is tight if it is minimal with respect to a lower bound for the number of points. To obtain the results, Bajnok [2, Proposition 14] essentially used the idea of the Sobolev theorem for $B_{n}$-invariant Euclidean designs, though he did not offer the name of Sobolev. It seems that some researchers in combinatorics and related areas do not fully recognized the Sobolev theorem [3].

In this paper we find some observations on invariant cubature formulas in connection with the Sobolev theorem. In Section 2 we explain Sobolev's invariant theory in detail. We also explain some basic facts related to Euclidean designs, e.g., a theorem of Neumaier and Seidel [20] which is well known in algebra and combinatorics. In Section 3 we give an alternative proof of famous theorems by $\mathrm{Xu}$ [28, Theorem 1.1, Theorem 1.2] on necessary and sufficient conditions for the existence of cubature formulas with radial symmetry. The original proof by $\mathrm{Xu}$ requires some tedious calculations and technical tools in numerical analysis like, Gaussian-Lobatto quadrature, Gaussian-Radau quadrature. Eventually it is long, and researchers in other areas may not be familiar with his proof. Whereas, our new proof is short and simple compared to the original proof. Moreover it gives a general interpretation of the analytically-written conditions of Xu's theorems, and so will be readable and acceptable for researchers not only in analysis, but also in other areas like algebra and combinatorics. In Section 4 we extend the theorem of Neumaier and Seidel to invariant Euclidean designs, and thereby classify tight Euclidean designs obtained from unions of the orbits of the corner vectors. This classification generalizes the result of Bajnok for other finite reflection groups.

\section{Preliminaries}

Let $\operatorname{Hom}_{l}\left(\mathbb{R}^{n}\right)$ be the linear space of all real homogeneous polynomials of total degree $l$ in $n$ variables. Let $\mathcal{P}_{l}\left(\mathbb{R}^{n}\right)=\sum_{i=0}^{l} \operatorname{Hom}_{i}\left(\mathbb{R}^{n}\right), \mathcal{P}_{l}^{*}\left(\mathbb{R}^{n}\right)=\sum_{i=0}^{\lfloor l / 2\rfloor} \operatorname{Hom}_{l-2 i}\left(\mathbb{R}^{n}\right)$. We denote by $\operatorname{Harm}_{l}\left(\mathbb{R}^{n}\right)$ the subspace of $\mathcal{P}_{l}\left(\mathbb{R}^{n}\right)$ of harmonic homogeneous polynomials of degree $l$. Let $\mathcal{P}_{l}(A), \mathcal{P}_{l}^{*}(A)$ be the space of functions which are the restrictions of the corresponding polynomials to $A \subset \mathbb{R}^{n}$.

Let $G$ be a finite subgroup of the orthogonal group in $\mathbb{R}^{n}$ and $f \in \mathcal{P}_{l}\left(\mathbb{R}^{n}\right)$. We consider the action of $\sigma \in G$ on $f$ as follows:

$$
(\sigma f)(x)=f\left(x^{\sigma^{-1}}\right), \quad x \in \mathbb{R}^{n} .
$$


A polynomial $f$ is said to be $G$-invariant if it satisfies that

$$
\sigma f=f, \quad \forall \sigma \in G .
$$

We denote by $\mathcal{P}_{l}\left(\mathbb{R}^{n}\right)^{G}, \operatorname{Harm}_{l}\left(\mathbb{R}^{n}\right)^{G}$ the set of $G$-invariant polynomials in $\mathcal{P}_{l}\left(\mathbb{R}^{n}\right)$, $\operatorname{Harm}_{l}\left(\mathbb{R}^{n}\right)$ respectively.

A cubature formula (1.1) is said to be invariant under $G$, or $G$-invariant if the domain $\Omega$ and measure $\mu$ of the integral are invariant under $G$ and the set of points is the union of $G$-orbits and to each point of the same orbit an equal weight is assigned. The following is known as the Sobolev theorem.

Theorem 2.1 ([25]). With the above set up, a G-invariant cubature formula is of degree $t$ if and only if it is exact for every polynomial $f \in \mathcal{P}_{t}\left(\mathbb{R}^{n}\right)^{G}$.

The Sobolev theorem is widely accepted by the cubature community in analysis and related areas: In particular Russian mathematicians in analysis have developed the Sobolev theorem and employed it to construct many cubature formulas; for instance see [19, 24]. Xu [28] presented beautiful theorems on the existence and structure of cubature formulas for radially symmetric integrals. In section 3 we review his theorems in detail and give a short proof using the Sobolev theorem.

Next let us explain a combinatorial object called Euclidean design which was introduced by Neumaier and Seidel 20 . Let $X$ be a finite set in $\mathbb{R}^{n}$. Let $r_{1}, r_{2}, \cdots, r_{p}$ be the norms of the vectors in $X$. For $i=1,2, \cdots, p$ we denote by $S_{i}^{n-1}$ the sphere of radius $r_{i}$ centered at the origin, namely, $S_{i}^{n-1}=\left\{x \in \mathbb{R}^{n} \mid\|x\|=r_{i}\right\}$, and let $X_{i}=X \cap S_{i}^{n-1}$. The set $X$ is said to be supported by $p$ concentric spheres $S=\bigcup_{i=1}^{p} S_{i}^{n-1}$. To each $S_{i}$ we assign the surface measure $\rho_{i}$. Let $\left|S_{i}^{n-1}\right|=\int_{S_{i}^{n-1}} \mathrm{~d} \rho_{i}(x)$, with the convention that $\frac{1}{\left|S_{i}^{n-1}\right|} \int_{S_{i}^{n-1}} f(x) \mathrm{d} \rho_{i}(x)=f(0)$ if $S_{i}^{n-1}=\{0\}$.

Definition 2.2. With the same notations as in the above paragraph, we say $X$ is a Euclidean $t$-design supported by $S$ if there exists a positive weight function $w(x)$ on $X$ such that

$$
\sum_{i=1}^{p} \frac{\sum_{x \in X_{i}} w(x)}{\left|S_{i}^{n-1}\right|} \int_{S_{i}^{n-1}} f(x) \mathrm{d} \rho_{i}(x)=\sum_{x \in X} w(x) f(x)
$$

for every polynomial $f \in \mathcal{P}_{t}(S)$.

We can regard a Euclidean design as a cubature formula on some concentric spheres. Conversely a cubature formula for a class of integral with some symmetry is a Euclidean design (cf. [15, Lemma 3.1]). The following theorem by Neumaier and Seidel is well known in algebra and combinatorics.

Theorem 2.3 (20]). With the same notations as in Definition 2.2, the following are equivalent:

(i) $X$ is a Euclidean $t$-design with a weight function $w$.

(ii) $\sum_{x \in X} w(x) f(x)=0$ for every $f \in\|x\|^{2 j} \operatorname{Harm}_{l}\left(\mathbb{R}^{n}\right)$ with $1 \leq l \leq t, 0 \leq$ $j \leq\left\lfloor\frac{t-l}{2}\right\rfloor$.

In Section 4 we give a stronger theorem than Theorem 2.3 for invariant Euclidean designs, especially for researchers in algebra and combinatorics.

Define $p^{\prime}=p-\varepsilon_{S}$, where $\varepsilon_{S}=1$ if $0 \in S$, and $\varepsilon_{S}=0$ otherwise. The dimensions of $\mathcal{P}_{l}(S)$ and $\mathcal{P}_{l}^{*}(S)$ are well known. 
Theorem 2.4 ([11, 13]). Let $S \subset \mathbb{R}^{n}$.
(1) $\operatorname{dim} \mathcal{P}_{l}(S)=\left\{\begin{array}{l}\varepsilon_{S}+\sum_{i=0}^{2 p^{\prime}-1}\left(\begin{array}{c}n+l-i-1 \\ n-1\end{array}\right) \\ \operatorname{dim} \mathcal{P}_{l}\left(\mathbb{R}^{n}\right)=\left(\begin{array}{c}n+l \\ l\end{array}\right)\end{array}\right.$
if $l \geq 2 p^{\prime}$ if $l \leq 2 p^{\prime}-1$.
(2) $\operatorname{dim} \mathcal{P}_{l}^{*}(S)=\left\{\begin{array}{l}\varepsilon_{S}+\sum_{i=0}^{p^{\prime}-1}\left(\begin{array}{c}n+l-2 i-1 \\ n-1\end{array}\right) \\ \sum_{i=0}^{p^{\prime}-1}\left(\begin{array}{c}n+l-2 i-1 \\ n-1\end{array}\right) \\ \operatorname{dim} \mathcal{P}_{l}^{*}\left(\mathbb{R}^{n}\right)=\sum_{i=0}^{\left\lfloor\frac{l}{2}\right\rfloor}\left(\begin{array}{c}n+l-2 i-1 \\ n-1\end{array}\right)\end{array}\right.$
if $l$ is even, $l \geq 2 p^{\prime}$,
if $l$ is odd, $l \geq 2 p^{\prime}$,
if $l \leq 2 p^{\prime}-1$.

The following lower bounds are known as the Fisher-type inequality for the size of a Euclidean design [6, 11, 17, 18; the latter one is also called the Möller bound.

Theorem 2.5. (1) Let $X$ be a Euclidean 2e-design supported by $S$. Then,

$$
|X| \geq \operatorname{dim} \mathcal{P}_{e}(S) \text {. }
$$

(2) Let $X$ be a Euclidean (2e-1)-design supported by $S$. Then,

$$
|X| \geq \begin{cases}2 \operatorname{dim} \mathcal{P}_{e-1}^{*}(S)-1 & \text { if } e \text { is odd and } 0 \in X, \\ 2 \operatorname{dim} \mathcal{P}_{e-1}^{*}(S) & \text { otherwise. }\end{cases}
$$

A Euclidean $t$-design is said to be tight if the equality holds in one of the bounds in Theorem 2.5

Hereafter we assume $G$ is a finite irreducible reflection group in $\mathbb{R}^{n}$. It is known that finite irreducible reflection groups are classified completely [9]. Let integers $1=m_{1} \leq m_{2} \leq \cdots \leq m_{n}$ be the exponents of $G$ (see [9, Ch.V, $\left.\S 6\right]$ ).

Theorem 2.6 (14). Let $G$ be a finite irreducible reflection group. Let $q_{i}$ be the dimension of $\operatorname{Harm}_{i}\left(\mathbb{R}^{n}\right)^{G}$. Then,

$$
\sum_{i=0}^{\infty} q_{i} \lambda^{i}=\prod_{i=2}^{n} \frac{1}{1-\lambda^{1+m_{i}}}
$$

Note that for any $x \in \mathbb{R}^{n}$, the orbit $x^{G}$ is a spherical $m_{2}$-design in $S^{n-1}$ [14].

Let $\alpha_{1}, \alpha_{2}, \ldots, \alpha_{n}$ be the fundamental roots of a reflection group $G$. The corner vectors $v_{1}, v_{2}, \ldots, v_{n}$ are defined by $v_{i} \perp \alpha_{j}$ if and only if $i \neq j$. We may assume $\left\|v_{k}\right\|=1$. We consider the set

$$
\mathcal{X}(G, J)=\bigcup_{k \in J} r_{k} v_{k}^{G},
$$

where $J \subset\{1,2, \ldots, n\}$ and $r_{k}>0$. Let $R$ denote the set of $r_{k}$.

Bajnok [2] found new tight Euclidean designs in $\mathcal{X}\left(B_{n}, J\right)$. In Section 4, using the theory of $G$-invariant harmonic polynomials, we extend the method of Bajnok to other reflection groups $G$, and classify the tight Euclidean designs obtained from $\mathcal{X}(G, J)$.

\section{XU'S THEOREM}

Let $\Omega=\left\{(x, y) \in \mathbb{R}^{2} \mid 0 \leq \sqrt{x^{2}+y^{2}}<\infty\right\}$. Let $W$ be a nonnegative weight function on $[0, \infty)$ with finite moments. We consider the radial weight function defined by $W\left(\sqrt{x^{2}+y^{2}}\right)$ on $\Omega$. The following integral is said to be radially symmetric 
or circularly symmetric:

$$
\begin{aligned}
\mathcal{I}[f] & =\int_{\Omega} f(x, y) W\left(\sqrt{x^{2}+y^{2}}\right) \mathrm{d} x \mathrm{~d} y \\
& =\int_{0}^{\infty}\left(\int_{0}^{2 \pi} f(r \cos \theta, r \sin \theta) \mathrm{d} \theta\right) r W(r) \mathrm{d} r .
\end{aligned}
$$

To generalize a famous theorem by Verlinden and Cools 27 on the existence of cubature formula for radially symmetric integral, Xu proved the following theorem:

Theorem 3.1. ([28, Theorem 2.1, Theorem 2.2]). (i) Let

$$
\begin{aligned}
\mathcal{I}_{2 m}(f)= & \frac{\pi}{m+1} \sum_{i=1}^{m} \lambda_{i} \sum_{j=0}^{2 m+1} f\left(r_{i} \cos \frac{\left(2 j+\sigma_{i}\right) \pi}{2 m+2}, r_{i} \sin \frac{\left(2 j+\sigma_{i}\right) \pi}{2 m+2}\right), \\
\mathcal{I}_{2 m+1}(f)= & \lambda_{0} f(0,0)+ \\
& \frac{\pi}{m+2} \sum_{i=1}^{m} \lambda_{i} \sum_{j=0}^{2 m+3} f\left(r_{i} \cos \frac{\left(2 j+\sigma_{i}\right) \pi}{2 m+4}, r_{i} \sin \frac{\left(2 j+\sigma_{i}\right) \pi}{2 m+4}\right),
\end{aligned}
$$

where $\sigma_{i}$ takes the value 0 if $m+i$ is even and the value 1 if $m+i$ is odd. Then, $\mathcal{I}_{n}$ forms a cubature formula of degree $2 n-1$ for $\mathcal{I}$ if and only if the following two types of conditions are satisfied:

$$
\begin{aligned}
\int_{0}^{\infty} r^{2 j+1} W(r) \mathrm{d} r & =\sum_{i=1}^{\lfloor n / 2\rfloor} \lambda_{i} r_{i}^{2 j}, \quad j=1, \cdots, n-1, \\
\sum_{i=1}^{\lfloor n / 2\rfloor} \lambda_{i} r_{i}^{2 j}(-1)^{i} & =0, \quad j=\lfloor(n+3) / 2\rfloor, \cdots, n-1 .
\end{aligned}
$$

(ii) With the same symbol $\sigma_{i}$ as in (i), let

$$
\mathcal{I}_{n}(f)=\frac{2 \pi}{m+1} \sum_{i=1}^{\lfloor(n+2) / 2\rfloor} \lambda_{i} \sum_{j=0}^{2 m} f\left(r_{i} \cos \frac{\left(2 j+\sigma_{i}\right) \pi}{2 m+1}, r_{i} \sin \frac{\left(2 j+\sigma_{i}\right) \pi}{2 m+1}\right),
$$

where $n=2 m-1$ or $2 m$. Then, $\mathcal{I}_{n}$ forms a cubature formula of degree $2 n$ for $\mathcal{I}$ if and only if the following two types of conditions are satisfied:

$$
\begin{gathered}
\int_{0}^{\infty} r^{2 j+1} W(r) \mathrm{d} r=\sum_{i=1}^{\lfloor(n+2) / 2\rfloor} \lambda_{i} r_{i}^{2 j}, \quad j=1, \cdots, n, \\
\sum_{i=1}^{\lfloor(n+2) / 2\rfloor} \lambda_{i} r_{i}^{2 j}(-1)^{i}=0, \quad j=\lfloor(n+1) / 2\rfloor, \cdots, n-1 .
\end{gathered}
$$

The aim of this section is to give a proof of Theorem 3.1 different from the original one by $\mathrm{Xu}$ :

Proof of Theorem 3.1. Let $D_{\ell}$ be the dihedral group of order $2 \ell$ and $f\left(x_{1}, x_{2}\right)$ be a $D_{\ell}$-invariant polynomial. Using the polar coordinate system, we let $f\left(x_{1}, x_{2}\right)=$ $f(r \cos \theta, r \sin \theta)$. It is shown that $f$ can be represented as a polynomial in two variables $r^{2}, r^{\ell} \cos \ell \theta$. We now consider the case where $n=2 m$ in (i); the reader will easily see that the same argument as below works in the remaining cases. The conditions (3.2), (3.3) respectively mean to substitute the monomials $r^{2}, \cdots, r^{2 n-2}$ 
and $r^{n+2} \cos (n+2) \theta, r^{n+4} \cos (n+2) \theta, \cdots, r^{2 n-2} \cos (n+2) \theta$ into (3.1). Thus the result follows by Theorem 2.1

Remark 3.2. (i) Xu's original proof of Theorem 3.1 is about 2 pages long only in the case of (i) with $n=2 \mathrm{~m}$. With this in mind, we tried a short proof using the Sobolev theorem. An advantage of our proof is the simplicity. Namely, the Sobolev theorem is the only advanced tool used in our proof, whereas, Xu's proof requires some technical and advanced tools in numerical analysis like, GaussianLobatto quadrature, Gaussian-Radau quadrature. The proof by $\mathrm{Xu}$ also requires some tedious calculations. Eventually our proof is shorter and simpler than the original one. Another advantage of our proof: The conditions (3.2), (3.3) (or (3.4), (3.5) ) are considerably analytic, and so researchers in other fields like combinatorics and algebra will not be familiar with them. Whereas, our new proof gives a general interpretation of the above analytic conditions, and will promise researchers in these areas to enjoy Theorem 3.1 well. The authors hope that researchers in many different fields know the importance of Theorem 3.1 and will be more interested in classical theories of cubature formulas developed in numerical analysis. (ii) Bajnok [1, Theorem 9] found a tight Euclidean $t$-design of $\mathbb{R}^{2}$ which has the same structure of points as Xu's formula, as a generalization of a tight 4-design by Bannai and Bannai [4. To do this, he implicitly used the same idea as in Theorem 3.1] for instance Eq. (10) in his paper corresponds to Eq. (3.3) (or Eq. (3.5) ) of our paper.

\section{Orbits OF A REFLECTION GROUP AS EUCLIDEAN DESIGNS}

In this section we classify the tight Euclidean designs obtained from $\mathcal{X}(G, J)$ for a finite irreducible reflection group $G$. A finite set $X \subset \mathbb{R}^{d}$ is said to be antipodal if $X=-X$. A tight Euclidean $2 e$-design has a weight function which is constant on each $X_{i}$ [4], and so does an antipodal tight Euclidean (2e-1)-design [8]. Throughout this section we assume a weight function is constant on each $G$-orbit.

First, we look at a stronger theorem than Theorem 2.3 for $G$-invariant Euclidean designs. A Euclidean $t$-design $X$ is said to be $G$-invariant if $X$ is a union of $G$-orbits and to each point of the same orbit an equal weight is assigned.

Lemma 4.1. Let $G$ be a subgroup of $O\left(\mathbb{R}^{n}\right)$. Let $f$ be a $G$-invariant polynomial and $x^{G}$ be a $G$-orbit. Then, $f(y)=f(z)$ for any $y, z \in x^{G}$.

Proof. Straightforward.

Let $|G|$ be the order of a group $G$.

Theorem 4.2. Let $G$ be a subgroup of $O\left(\mathbb{R}^{n}\right)$. Let $X=\cup_{k=1}^{M} r_{k} x_{k}^{G}$, where $x_{k} \in$ $S^{n-1}$ and $r_{k}>0$. The following are equivalent:

(1) $X$ is a G-invariant Euclidean t-design.

(2) $\sum_{x \in X} w(x)\|x\|^{2 j} \varphi(x)=0$ for any $\varphi \in \operatorname{Harm}_{l}\left(\mathbb{R}^{n}\right)^{G}$ with $1 \leq l \leq t, 0 \leq$ $j \leq\left\lfloor\frac{t-l}{2}\right\rfloor$.

Proof. For $f \in \operatorname{Harm}_{l}\left(\mathbb{R}^{d}\right)$, the polynomial

$$
\varphi(\xi)=\frac{1}{|G|} \sum_{g \in G} f\left(\xi^{g}\right)
$$


is an element of $\operatorname{Harm}_{l}\left(\mathbb{R}^{d}\right)^{G}$. Let $w(x)=w_{k}$ for every $x \in x_{k}^{G}$. By Lemma 4.1 for any $f \in \operatorname{Harm}_{l}\left(\mathbb{R}^{d}\right)$, we have

$$
\begin{aligned}
\sum_{x \in X} w(x)\|x\|^{2 j} f(x) & =\sum_{k=1}^{M} w_{k} r_{k}^{2 j} \sum_{x \in x_{k}^{G}} f\left(r_{k} x\right) \\
& =\sum_{k=1}^{M} \frac{w_{k} r_{k}^{2 j+l}\left|x_{k}^{G}\right|}{|G|} \sum_{g \in G} f\left(x_{k}^{g}\right) \\
& =\sum_{k=1}^{M} w_{k} r_{k}^{2 j+l}\left|x_{k}^{G}\right| \varphi\left(x_{k}\right) \\
& =\sum_{k=1}^{M} w_{k} r_{k}^{2 j} \sum_{x \in x_{k}^{G}} \varphi\left(r_{k} x\right) \\
& =\sum_{x \in X} w(x) \|\left. x\right|^{2 j} \varphi(x) .
\end{aligned}
$$

The result thus follows by Theorem 2.3

Remark 4.3. The radii $r_{k}$ are not necessarily mutually distinct in Theorem 4.2 Goethals and Seidel 14 stated Theorem 2.1 for spherical designs. Theorem 4.2 with all $r_{k}=1$ means the theorem of $G$-invariant spherical designs. The approach of using the orbits under subgroups of $O\left(\mathbb{R}^{d}\right)$ has long been considered [5]. Theorem 4.2 reduces the computational cost to check the strength of a $G$-invariant Euclidean design less than using Theorem 2.3.

From now on, let $G$ be a finite irreducible reflection group, and $v_{k}$ be a corner vector. Put $N_{k}=\left|v_{k}^{G}\right|$. The following is immediate by Lemma 4.1 and Theorem 4.2 .

Corollary 4.4. $\mathcal{X}(G, J)$ is a Euclidean $t$-design if and only if there exist $w_{k}>0$ and $r_{k}>0$ such that the equation $\sum_{k \in J} w_{k} r_{k}^{2 j+i} N_{k} f\left(v_{k}\right)=0$ holds for any $f \in$ $\operatorname{Harm}_{i}\left(\mathbb{R}^{d}\right)^{G}$, where $i, j$ are positive integers satisfying $1 \leq 2 j+i \leq t$.

The dimension of $\operatorname{Harm}_{i}\left(\mathbb{R}^{d}\right)^{G}$ is clear by Theorem 2.6. We can determine the basis of $\operatorname{Harm}_{i}\left(\mathbb{R}^{d}\right)^{G}$ by harmonic polynomials $f$ satisfying $f\left(x^{g}\right)=f(x)$ for each generator $g$ of $G$. By the basis of $\operatorname{Harm}_{i}\left(\mathbb{R}^{d}\right)^{G}$ and Corollary 4.4, we know a necessary and sufficient condition for $\mathcal{X}(G, J)$ to be a Euclidean $t$-design. Bajnok 2 found an explicit such condition for the group $B_{n}$ by using Corollary 4.4. For other groups, it is possible to give the conditions, but the statements are not simple. Therefore we do not write them in the present paper.

Now, let us classify the tight Euclidean design obtained from $\mathcal{X}(G, J)$. For each group, we determine the possible maximum strength of $\mathcal{X}(G, J)$ for any $J$ and radii $R$. Since the cardinality of $v_{k}^{G}$ is easily calculated, we can check whether the total size of a union of several orbits attains the fisher type inequality. For the set attaining the bound, we give its maximum strength by Corollary 4.4

Hereafter let $e_{i} \in \mathbb{R}^{n}$ be the row vector whose $i$-th entry is 1 and other entries are 0 . Let $S_{n}$ be the symmetric group. Define

$$
\operatorname{sym}(f):=\frac{1}{\left|\left(S_{n}\right)_{f}\right|} \sum_{g \in S_{n}} f\left(x^{g}\right),
$$


where $\left(S_{n}\right)_{f}:=\left\{g \in S_{n} \mid f\left(x^{g}\right)=f(x)\right\}$.

\subsection{Group $A_{n}$. \\ Dynkin diagram}

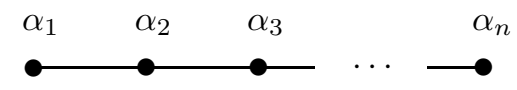

\section{Exponents}

$1,2, \ldots, n$

Fundamental roots

$\alpha_{i}:=e_{i}-e_{i+1}$ for $1 \leq i \leq n-1 . \alpha_{n}:=[a, a, \ldots, a, b]$ where $a=(-1+\sqrt{n+1}) / n$ and $b=(n-1+\sqrt{n+1}) / n$.

\section{Corner Vectors}

$v_{k}=\left[c_{k}, \ldots, c_{k}, d_{k}, \ldots, d_{k}\right]$ whose first $k$ coordinates are equal to $c_{k}$, and last $n-k$ coordinates are equal to $d_{k}$, where

$$
c_{k}=\frac{n+1-k+\sqrt{n+1}}{\sqrt{k(n+1-k)(n+2+2 \sqrt{n+1})}}, \quad d_{k}=\frac{-k}{\sqrt{k(n+1-k)(n+2+2 \sqrt{n+1})}} .
$$

\section{Reflection group}

The reflection group $A_{n} \subset O\left(\mathbb{R}^{n}\right)$ is generated by the following:

$$
\begin{gathered}
r\left(\alpha_{i}\right)=\left[{ }^{t} e_{1}, \cdots{ }^{t} e_{i-1},{ }^{t} e_{i+1},{ }^{t} e_{i},{ }^{t} e_{i+2}, \cdots,{ }^{t} e_{n}\right] \text { for } 1 \leq i \leq n-1, \\
r\left(\alpha_{n}\right)=\left[\begin{array}{cc}
I_{n-1}-a^{2}\left({ }^{t} j j\right) & -a b^{t} j \\
-a b j & 1-b^{2}
\end{array}\right],
\end{gathered}
$$

where $I_{n}$ is the identity matrix of size $n$, and $j$ is the all-ones row vector.

\section{Orbits}

Let $U_{1}$ be the set of all vectors such that $k$ coordinates are equal to $c_{k}$, and other $n-k$ coordinates are equal to $d_{k}$. Let $U_{2}$ be the set of all vectors such that $k-1$ coordinates are equal to $c_{k}^{\prime}$, and other $n+1-k$ coordinates are equal to $d_{k}^{\prime}$, where

$$
c_{k}^{\prime}=\frac{n+1-k}{\sqrt{k(n+1-k)(n+2+2 \sqrt{n+1})}}, \quad d_{k}^{\prime}=\frac{-k-\sqrt{n+1}}{\sqrt{k(n+1-k)(n+2+2 \sqrt{n+1})}} .
$$

Then the orbit $v_{k}^{A_{n}}=U_{1} \cup U_{2}$. Furthermore, we have $N_{k}=\left(\begin{array}{c}n+1 \\ k\end{array}\right)$ and $v_{k}^{A_{n}}=$ $-v_{n+1-k}^{A_{n}}$.

\section{Harmonic Molien series}

$$
\frac{1}{\left(1-t^{3}\right)\left(1-t^{4}\right) \cdots\left(1-t^{n+1}\right)}= \begin{cases}1+t^{3}+t^{6}+\cdots, & \text { if } n=2, \\ 1+t^{3}+t^{4}+t^{6}+\cdots, & \text { if } n=3, \\ 1+t^{3}+t^{4}+t^{5}+\cdots, & \text { if } n \geq 4\end{cases}
$$

\section{$G$-invariant harmonic polynomials}

1. Degree 3.

Note that $\operatorname{dim} \operatorname{Harm}_{3}\left(\mathbb{R}^{n}\right)^{A_{n}}=1$ for any $n \geq 2 . \operatorname{Harm}_{3}\left(\mathbb{R}^{n}\right)^{A_{n}}$ is spanned by the following:

(i) $n=2$.

(ii) $n=3$.

$$
f_{3}=x_{1}^{3}-3 x_{1}^{2} x_{2}-3 x_{1} x_{2}^{2}+x_{2}^{3} .
$$

$$
f_{3}=\operatorname{sym}\left(x_{1}^{3}\right)-\frac{3}{2} \operatorname{sym}\left(x_{1} x_{2}^{2}\right)-\frac{3}{4} \operatorname{sym}\left(x_{1} x_{2} x_{3}\right) .
$$


(ii) $n \geq 4$.

$$
f_{3}=\operatorname{sym}\left(x_{1}^{3}\right)-\frac{3}{n-1} \operatorname{sym}\left(x_{1} x_{2}^{2}\right)+\frac{6(2-\sqrt{n+1})}{(n-1)(n-3)} \operatorname{sym}\left(x_{1} x_{2} x_{3}\right) .
$$

2. Degree 4 .

Note that $\operatorname{dim} \operatorname{Harm}_{4}\left(\mathbb{R}^{2}\right)^{A_{2}}=0$ and $\operatorname{dim} \operatorname{Harm}_{4}\left(\mathbb{R}^{n}\right)^{A_{n}}=1$ for any $n \geq 3$. The following are $S_{n}$-invariant harmonic polynomials:

$$
\begin{aligned}
& h_{4,1}=\operatorname{sym}\left(x_{1}^{4}\right)-\frac{6}{n-2} \operatorname{sym}\left(x_{1}^{2} x_{2}^{2}\right), \\
& h_{4,2}=\operatorname{sym}\left(x_{1} x_{2} x_{3} x_{4}\right), \\
& h_{4,3}=\operatorname{sym}\left(x_{1} x_{2}^{3}\right)-\frac{6}{n-2} \operatorname{sym}\left(x_{1} x_{2} x_{3}^{2}\right) .
\end{aligned}
$$

$\operatorname{Harm}_{4}\left(\mathbb{R}^{n}\right)^{A_{n}}$ is spanned by the following:

(i) $n=3$.

$$
f_{4}=h_{4,1}-\frac{20}{13} h_{4,3}
$$

(ii) $n \geq 4$.

$$
f_{4}=h_{4,1}+c_{4,2} h_{4,2}+c_{4,3} h_{4,3},
$$

where

$$
\begin{aligned}
& c_{4,2}=\frac{24(n+2)\left(n^{2}-5 n-12+4 \sqrt{n+1}\right)}{(n-1)(n-2)\left(n^{3}-2 n^{2}-15 n-16\right)}, \\
& c_{4,3}=-\frac{4(n+2)\left(n^{2}-2 n-7-(n-1) \sqrt{n+1}\right)}{(n-1)\left(n^{3}-2 n^{2}-15 n-16\right)} .
\end{aligned}
$$

3. Degree 5.

Note that $\operatorname{dim} \operatorname{Harm}_{5}\left(\mathbb{R}^{n}\right)^{A_{n}}=0$ for $n=2,3$, and $\operatorname{dim}^{\operatorname{Harm}_{5}}\left(\mathbb{R}^{n}\right)^{A_{n}}=1$ for any $n \geq 4$. The following are $S_{n}$-invariant harmonic polynomials:

$$
\begin{aligned}
& h_{5,1}=\operatorname{sym}\left(x_{1}^{5}\right)-\frac{10}{n-1} \operatorname{sym}\left(x_{1}^{2} x_{2}^{3}\right)+\frac{30}{(n-1)(n-2)} \operatorname{sym}\left(x_{1} x_{2}^{2} x_{3}^{2}\right), \\
& h_{5,2}=\operatorname{sym}\left(x_{1}^{5}\right)-\frac{10}{n-1} \operatorname{sym}\left(x_{1}^{2} x_{2}^{3}\right)+\frac{5}{n-1} \operatorname{sym}\left(x_{1} x_{2}^{4}\right), \\
& h_{5,3}=\operatorname{sym}\left(x_{1} x_{2} x_{3}^{3}\right)-\frac{9}{n-3} \operatorname{sym}\left(x_{1} x_{2} x_{3} x_{4}^{2}\right), \\
& h_{5,4}=\operatorname{sym}\left(x_{1} x_{2} x_{3} x_{4} x_{5}\right) .
\end{aligned}
$$

$\operatorname{Harm}_{4}\left(\mathbb{R}^{n}\right)^{A_{n}}$ is spanned by the following:

(i) $n=4$.

$$
f_{5}=h_{5,1}+\frac{17-20 \sqrt{5}}{58} h_{5,2}+\frac{10(18+\sqrt{5})}{87} h_{5,3}
$$

(ii) $n \geq 5$.

$$
f_{5}=h_{5,1}+c_{5,2} h_{5,2}+c_{5,3} h_{5,3}+c_{5,4} h_{5,4},
$$


where

$$
\begin{aligned}
& c_{5,2}=-\frac{2 n^{3}+5 n^{2}-21 n-90-n(n+6) \sqrt{n+1}}{4 n^{3}+3 n^{2}-60 n-180}, \\
& c_{5,3}=\frac{20\left(2 n^{3}+6 n^{2}-32 n-168+\left(n^{2}-8 n+12\right) \sqrt{n+1}\right)}{(n-1)(n-2)\left(4 n^{3}+3 n^{2}-60 n-180\right)}, \\
& c_{5,4}=-\frac{120(n+6)\left(n^{2}-11 n-78+\left(2 n^{2}-2 n+12\right) \sqrt{n+1}\right)}{(n-1)(n-2)(n-3)\left(4 n^{3}+3 n^{2}-60 n-180\right)} .
\end{aligned}
$$

\section{Substitute $v_{k}$ for $G$-invariant harmonic polynomials}

1. Degree 3 .

For $n=2, n \geq 4$,

$$
f_{3}\left(v_{k}\right)=-\frac{k-\frac{n+1}{2}}{\sqrt{k(n+1-k)}} \phi_{3}(n),
$$

where

$$
\phi_{3}(n)=\frac{2\left(n^{3}+3 n^{2}-12 n-16+\left(3 n^{2}-4 n-16\right) \sqrt{n+1}\right)}{(n-1)(n-3)(n+2+2 \sqrt{n+1})^{\frac{3}{2}}} .
$$

For $n=3$,

$$
f_{3}\left(v_{1}\right)=\frac{729}{4}, \quad f_{3}\left(v_{2}\right)=0, \quad f_{3}\left(v_{3}\right)=-\frac{729}{4} .
$$

2. Degree 4 .

For $n \geq 3$,

$$
f_{4}\left(v_{k}\right)=\frac{(k-\alpha)(k-\beta)}{k(n+1-k)} \phi_{4}(n),
$$

where

$$
\begin{aligned}
& \phi_{4}(n) \\
& =\frac{6(n+1)\left(n^{5}+7 n^{4}-24 n^{3}-160 n^{2}-256 n-128+4\left(n^{4}-20 n^{2}-48 n-32\right) \sqrt{n+1}\right)}{(n-1)(n-2)\left(n^{3}-2 n^{2}-15 n-16\right)(n+2+2 \sqrt{n+1})^{2}}, \\
& \alpha=\frac{n+1}{2}-\frac{\sqrt{3\left(n^{2}-1\right)}}{6}, \quad \beta=\frac{n+1}{2}+\frac{\sqrt{3\left(n^{2}-1\right)}}{6} .
\end{aligned}
$$

3. Degree 5 .

For $n \geq 4$,

$$
f_{5}\left(v_{k}\right)=-\frac{\left(k-\frac{n+1}{2}\right)\left(k-\alpha^{\prime}\right)\left(k-\beta^{\prime}\right)}{(k(n+1-k))^{\frac{3}{2}}} \phi_{5}(n),
$$

where

$$
\begin{aligned}
\phi_{5}(n)= & \frac{24(n+1)\left(2 n^{6}+31 n^{5}+50 n^{4}-448 n^{3}-2144 n^{2}-3200 n-1536\right)}{(n-1)(n-2)(n-3)\left(4 n^{3}+3 n^{2}-60 n-180\right)(n+2+2 \sqrt{n+1})^{\frac{5}{2}}} \\
& +\frac{24(n+1)\left(11 n^{5}+50 n^{4}-96 n^{3}-1120 n^{2}-2432 n-1536\right) \sqrt{n+1}}{(n-1)(n-2)(n-3)\left(4 n^{3}+3 n^{2}-60 n-180\right)(n+2+2 \sqrt{n+1})^{\frac{5}{2}}}, \\
\alpha^{\prime}= & \frac{n+1}{2}-\frac{\sqrt{3(n+1)(2 n-3)}}{6}, \quad \beta^{\prime}=\frac{n+1}{2}+\frac{\sqrt{3(n+1)(2 n-3)}}{6} .
\end{aligned}
$$

Theorem 4.5. There is no choice of $J, R$ and $w$ for which $\left(\mathcal{X}\left(A_{n}, J\right), w\right)$ is a Euclidean 6-design. 
NOTE ON CUBATURE FORMULAE AND DESIGNS OBTAINED FROM GROUP ORBITS 11

Proof. The polynomial of degree 6

$$
f\left(x_{1}, x_{2}, \ldots, x_{n}\right)=\operatorname{sym}\left(x_{1} x_{2}^{5}\right)-\frac{10}{3} \operatorname{sym}\left(x_{1}^{3} x_{2}^{3}\right)
$$

is harmonic for any $n \geq 2$. We can calculate

$$
\sum_{x \in v_{k}^{A_{n}}} f(x)=g_{1}(n, k)\left(k(k-n-1)\left(4 k^{2}-4(n+1) k+n^{2}+5 n+4\right) g_{2}(n)+g_{3}(n)\right),
$$

where

$$
\begin{aligned}
& g_{1}(n, k)=\frac{n(n+1)}{3 k^{3}(n+1-k)^{3}(n+2+2 \sqrt{n+1})^{3}}\left(\begin{array}{c}
n-1 \\
k-1
\end{array}\right), \\
& g_{2}(n)=5\left(n^{2}+11 n+12+(6 n+12) \sqrt{n+1}\right), \\
& g_{3}(n)=(n+1)^{2}(n+2)\left(2 n^{2}+28 n+30+(15 n+30) \sqrt{n+1}\right) .
\end{aligned}
$$

Note $g_{1}(n, k)>0$ for $1 \leq k \leq n$. Define

$$
F(k)=k(k-n-1)\left(4 k^{2}-4(n+1) k+n^{2}+5 n+4\right) g_{2}(n)+g_{3}(n) .
$$

For a fixed $n$, we prove $F(k)<0$ for $1 \leq k \leq n$. We have

$$
\frac{d}{d k} F(k)=16\left(k-\frac{n+1}{2}\right)\left(k-\alpha^{\prime \prime}\right)\left(k-\beta^{\prime \prime}\right) g_{2}(n)
$$

where

$$
\alpha^{\prime \prime}=\frac{n+1}{2}-\frac{\sqrt{2(n+1)(n-2)}}{4}, \quad \beta^{\prime \prime}=\frac{n+1}{2}+\frac{\sqrt{2(n+1)(n-2)}}{4} .
$$

If $F(1)=F(n)<0, F((n+1) / 2)<0$, and $F\left(\alpha^{\prime \prime}\right)=F\left(\beta^{\prime \prime}\right)<0$, then $F(k)<0$ for all $1 \leq k \leq n$. Indeed for $n \geq 2$,

$$
\begin{aligned}
& \begin{aligned}
& F(1)=F(n) \\
&=-(n-1)\left(3 n^{4}+27 n^{3}+10 n^{2}+26 n+60+\left(15 n^{3}+15 n^{2}+60\right) \sqrt{n+1}\right)<0, \\
& \begin{aligned}
F\left(\alpha^{\prime \prime}\right) & =F\left(\beta^{\prime \prime}\right) \\
& =-\frac{1}{16} n(n+1)^{2}\left(5 n^{3}+63 n^{2}+68 n-16+\left(30 n^{2}+60 n\right) \sqrt{n+1}\right)<0,
\end{aligned} \\
& F\left(\frac{n+1}{2}\right)=-\frac{1}{4}(n-1)(n+1)^{2}\left(7 n^{2}+59 n+60+(30 n+60) \sqrt{n+1}\right)<0 .
\end{aligned}
\end{aligned}
$$

Therefore $\sum_{k \in J} \sum_{x \in v_{k}^{A_{n}}} w_{k} r_{k} f(x)<0$ for any $J, R$ and $w$.

Theorem 4.6. $\mathcal{X}\left(A_{n}, J\right)$ is not a tight Euclidean $t$-design except for the sets in Table 1.

Proof. We prove only the classification of tight Euclidean 4-designs on two concentric spheres obtained from $\mathcal{X}\left(A_{n}, J\right)$. The other cases can be proved by a similar way.

$f_{3}\left(v_{k}\right)$ and $\phi_{3}(n)$ are defined as above. Since $\phi_{3}(n)>0$ for $n>1, f_{3}\left(v_{k}\right)=0$ if and only if $n \equiv 1(\bmod 2)$ and $k=(n+1) / 2$. Clearly $f_{3}\left(v_{k}\right)>0$ for $k<(n+1) / 2$, and $f_{3}\left(v_{k}\right)<0$ for $k>(n+1) / 2$. Therefore $J$ must contain $k_{1}$ and $k_{2}$ such that $k_{1}<(n+1) / 2<k_{2}$ by Corollary 4.4 .

The size of a tight Euclidean 4-design on two concentric spheres is $(n+1)(n+2) / 2$. By noting that $(n+1)(n+2) / 2<N_{3}=N_{n-2}$ for $n>5$, we can determine $J=\{1, n-1\}$, (or equivalently $J=\{2, n\}$ ) for any $n>2$, or $J=\{1,2\}$ for $n=2$. 


\begin{tabular}{|c|c|c|c|c|c|}
\hline$|R|$ & $t$ & $n$ & $J$ & $r_{i}$ & $w_{i}$ \\
\hline 1 & 2 & any & $\{1\}$ & $r_{1}=1$ & $w_{1}=1$ \\
& 2 & any & $\{n\}$ & $r_{n}=1$ & $w_{n}=1$ \\
& 3 & 3 & $\{2\}$ & $r_{2}=1$ & $w_{2}=1$ \\
& 5 & 2 & $\{1,2\}$ & $r_{1}=r_{2}=1$ & $w_{1}=w_{2}=1$ \\
& 5 & 7 & $\{2,6\}$ & $r_{2}=r_{6}=1$ & $w_{2}=w_{6}=1$ \\
\hline 2 & 4 & 2 & $\{1,2\}$ & $r_{1}=1, r_{2} \neq 1$ & $w_{1}=1, w_{2}=\frac{1}{r_{2}^{3}}$ \\
& 4 & 4 & $\{1,3\}$ & $r_{1}=1, r_{3}=\frac{1}{\sqrt{6}}$ & $w_{1}=1, w_{3}=27$ \\
& 4 & 4 & $\{2,4\}$ & $r_{4}=1, r_{2}=\frac{1}{\sqrt{6}}$ & $w_{4}=1, w_{2}=27$ \\
& 4 & 5 & $\{1,4\}$ & $r_{1}=1, r_{4}=\sqrt{\frac{8}{5}}$ & $w_{1}=1, w_{4}=\frac{1}{2}$ \\
& 4 & 5 & $\{2,5\}$ & $r_{5}=1, r_{2}=\sqrt{\frac{8}{5}}$ & $w_{5}=1, w_{2}=\frac{1}{2}$ \\
& 4 & 6 & $\{1,5\}$ & $r_{1}=1, r_{5}=\sqrt{15}$ & $w_{1}=1, w_{5}=\frac{1}{81}$ \\
& 4 & 6 & $\{2,6\}$ & $r_{6}=1, r_{2}=\sqrt{15}$ & $w_{6}=1, w_{2}=\frac{1}{81}$ \\
& 5 & 3 & $\{1,2,3\}$ & $r_{1}=r_{3}=1, r_{2} \neq 1$ & $w_{1}=w_{3}=1, w_{2}=\frac{9}{8 r_{2}^{4}}$ \\
& 5 & 5 & $\{1,3,5\}$ & $r_{1}=r_{5}=1, r_{3} \neq 1$ & $w_{1}=w_{5}=1, w_{3}=\frac{27}{25 r_{2}^{4}}$ \\
\hline
\end{tabular}

TABLE 1. Tight Euclidean $t$-designs from $\mathcal{X}\left(A_{n}, J\right)$

For $n=2$, we can obtain tight Euclidean 4-designs on two concentric spheres as in Table 1.

$f_{4}\left(v_{k}\right), \phi_{4}(n), \alpha$, and $\beta$ are defined as above. Note that $\phi_{4}(n) \neq 0, \alpha>1$, and $\beta<n$ for any integer $n>2$. Therefore $1<\alpha<n-1<\beta$, (or equivalently $\alpha<2<\beta<n$ ) holds by Corollary 4.4. The integers satisfying the condition are only $n=4,5,6$. For $n=4,5,6$, we can obtain tight Euclidean 4-designs on two concentric spheres as in Table 1.

\subsection{Group $B_{n}$.}

\section{Dynkin diagram}

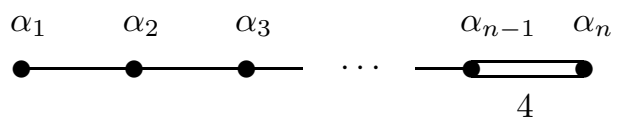

\section{Exponents}

$1,3, \ldots, 2 n-1$

\section{Fundamental roots}

$\alpha_{i}:=e_{i}-e_{i+1}$ for $1 \leq i \leq n-1$ and $\alpha_{n}:=\sqrt{2} e_{n}$.

\section{Corner Vectors}

$v_{k}=[1 / \sqrt{k}, \ldots, 1 / \sqrt{k}, 0, \ldots, 0]$, where $v_{k}$ has $k$ coordinates equal to $1 / \sqrt{k}$.

\section{Reflection group}

The reflection group $B_{n} \subset O\left(\mathbb{R}^{n}\right)$ is generated by the following:

$$
\begin{gathered}
r\left(\alpha_{i}\right)=\left[{ }^{t} e_{1}, \cdots{ }^{t} e_{i-1},{ }^{t} e_{i+1},{ }^{t} e_{i},{ }^{t} e_{i+2}, \cdots,{ }^{t} e_{n}\right] \text { for } 1 \leq i \leq n-1 \\
r\left(\alpha_{n}\right)=\left[{ }^{t} e_{1}, \cdots{ }^{t} e_{n-1},-{ }^{t} e_{n}\right]
\end{gathered}
$$

\section{Orbits}

The orbit $v_{k}^{B_{n}}$ is the set of vectors with exactly $k$ nonzero coordinates equal to $\pm 1 / \sqrt{k}$. Note that $v_{k}^{B_{n}}$ is antipodal and $N_{k}=2^{k}\left(\begin{array}{l}n \\ k\end{array}\right)$. 


\begin{tabular}{|c|c|c|c|c|c|}
\hline$|R|$ & $t$ & $n$ & $J$ & $r_{i}$ & $w_{i}$ \\
\hline 1 & 3 & any & $\{1\}$ & $r_{1}=1$ & $w_{1}=1$ \\
& 3 & 2 & $\{2\}$ & $r_{2}=1$ & $w_{2}=1$ \\
& 7 & 2 & $\{1,2\}$ & $r_{1}=r_{2}=1$ & $w_{1}=w_{2}=1$ \\
\hline 2 & 5 & 2 & $\{1,2\}$ & $r_{1}=1, r_{2} \neq 1$ & $w_{1}=1, w_{2}=\frac{1}{r_{2}^{4}}$ \\
& 5 & 3 & $\{1,3\}$ & $r_{1}=1, r_{3} \neq 1$ & $w_{1}=1, w_{3}=\frac{9}{8 r_{2}^{4}}$ \\
& 7 & 4 & $\{1,2,4\}$ & $r_{1}=r_{4}=1, r_{2} \neq 1$ & $w_{1}=w_{4}=1, w_{2}=\frac{1}{r_{2}^{6}}$ \\
\hline 3 & 7 & 3 & $\{1,2,3\}$ & $r_{1}=1, r_{2}=\sqrt{\frac{2 r_{3}^{2}}{5 r_{3}^{2}-3},\left(r_{i} \neq r_{j}\right)}$ & $w_{1}=1, w_{2}=\frac{4}{5 r_{2}^{6}}, w_{3}=\frac{27}{40 r_{3}^{6}}$ \\
\hline \multicolumn{5}{|c}{ TABLE 2. Tight Euclidean $t$-designs from $\mathcal{X}\left(B_{n}, J\right)$}
\end{tabular}

\section{Harmonic Molien series}

$$
\frac{1}{\left(1-t^{4}\right)\left(1-t^{6}\right) \cdots\left(1-t^{2 n}\right)}= \begin{cases}1+t^{4}+t^{8}+\cdots, & \text { if } n=2 \\ 1+t^{4}+t^{6}+\cdots, & \text { if } n \geq 3\end{cases}
$$

\section{$G$-invariant harmonic polynomials}

1. Degree 4.

Note that $\operatorname{dim}\left(\operatorname{Harm}_{4}\left(\mathbb{R}^{n}\right)^{B_{n}}\right)=1$ for any $n \geq 2$. The following is a $B_{n}$-invariant harmonic polynomial of degree 4 :

$$
f_{4}=\operatorname{sym}\left(x_{1}^{4}\right)-\frac{6}{n-1} \operatorname{sym}\left(x_{1}^{2} x_{2}^{2}\right) .
$$

2. Degree 6.

Note that $\operatorname{dim}\left(\operatorname{Harm}_{6}\left(\mathbb{R}^{2}\right)^{B_{2}}\right)=0$ and $\operatorname{dim}\left(\operatorname{Harm}_{6}\left(\mathbb{R}^{n}\right)^{B_{n}}\right)=1$ for any $n \geq 3$. The following is a $B_{n}$-invariant harmonic polynomials of degree 6 :

$$
f_{6}=\operatorname{sym}\left(x_{1}^{6}\right)-\frac{15}{n-1} \operatorname{sym}\left(x_{1}^{2} x_{2}^{4}\right)+\frac{180}{(n-1)(n-2)} \operatorname{sym}\left(x_{1}^{2} x_{2}^{2} x_{3}^{2}\right) .
$$

Substitute $v_{k}$ for $G$-invariant harmonic polynomials 1. Degree 4.

$$
f_{4}\left(v_{k}\right)=\frac{1}{k}\left(1-3 \frac{k-1}{n-1}\right)
$$

2. Degree 6 .

$$
f_{6}\left(v_{k}\right)=\frac{1}{k^{2}}\left(1-15 \frac{k-1}{n-1}+30 \frac{(k-1)(k-2)}{(n-1)(n-2)}\right) .
$$

Theorem $4.7([2])$. There is no choice of $R, J$, and $w$ for which $\left(\mathcal{X}\left(B_{n}, J\right), w\right)$ is a Euclidean 8-design.

Theorem $4.8([2]) . \mathcal{X}\left(B_{n}, J\right)$ is not a tight Euclidean $t$-design except for the sets in Table 2.

Remark 4.9. We can also prove Theorems 4.7 and 4.8 by the $B_{n}$-invariant harmonic polynomials. 


\subsection{Group $D_{n}$.}

Dynkin diagram

\section{Exponents}

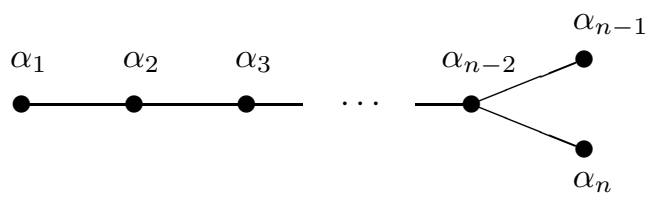

$1,3, \ldots, 2 n-3, n-1$

\section{Fundamental roots}

$\alpha_{i}:=e_{i}-e_{i+1}$ for $1 \leq i \leq n-1$ and $\alpha_{n}:=e_{n-1}+e_{n}$.

\section{Corner Vectors}

$v_{k}=[1 / \sqrt{k}, \ldots, 1 / \sqrt{k}, 0, \ldots, 0]$, where $v_{k}$ has $k$ coordinates equal to $1 / \sqrt{k}$ for $1 \leq$ $k \leq n-2 . v_{n-1}=[1 / \sqrt{n}, 1 / \sqrt{n}, \ldots, 1 / \sqrt{n},-1 / \sqrt{n}]$ and $v_{n}=[1 / \sqrt{n}, 1 / \sqrt{n}, \ldots, 1 / \sqrt{n}]$.

\section{Reflection group}

The reflection group $D_{n} \subset O\left(\mathbb{R}^{n}\right)$ is generated by the following:

$$
\begin{gathered}
r\left(\alpha_{i}\right)=\left[{ }^{t} e_{1}, \cdots{ }^{t} e_{i-1},{ }^{t} e_{i+1},{ }^{t} e_{i},{ }^{t} e_{i+2}, \cdots,{ }^{t} e_{n}\right] \text { for } 1 \leq i \leq n-1, \\
r\left(\alpha_{n}\right)=\left[{ }^{t} e_{1}, \cdots{ }^{t} e_{n-2},-{ }^{t} e_{n},-{ }^{t} e_{n-1}\right] .
\end{gathered}
$$

Orbits

For $1 \leq k \leq n-2, v_{k}^{D_{n}}=v_{k}^{B_{n}}$. The orbit $v_{n}^{D_{n}}$ (resp. $v_{n-1}^{D_{n}}$ ) consists of the vectors $\{ \pm 1 / \sqrt{n}\}^{n}$ with an even (resp. odd) number of negative coordinates. Note that $v_{n}^{D_{n}}=-v_{n-1}^{D_{n}}$ for odd $n$, and both $v_{n}^{D_{n}}$ and $v_{n-1}^{D_{n}}$ are antipodal for even $n$. Furthermore, $\left|N_{n-1}\right|=\left|N_{n}\right|=2^{n-1}$.

Harmonic Molien series

$\frac{1}{\left(1-t^{4}\right)\left(1-t^{6}\right) \cdots\left(1-t^{2 n-2}\right)\left(1-t^{n}\right)}= \begin{cases}1+2 t^{4}+t^{6}+3 t^{8}+\cdots, & \text { if } n=4, \\ 1+t^{4}+t^{5}+t^{6}+2 t^{8}+\cdots, & \text { if } n=5, \\ 1+t^{4}+2 t^{6}+2 t^{8}+\cdots, & \text { if } n=6, \\ 1+t^{4}+t^{6}+t^{7}+2 t^{8} \cdots, & \text { if } n=7, \\ 1+t^{4}+t^{6}+2 t^{8} \cdots, & \text { if } n \geq 8 .\end{cases}$

\section{$G$-invariant harmonic polynomials}

1. Degree 4.

Note that $\operatorname{dim}\left(\operatorname{Harm}_{4}\left(\mathbb{R}^{4}\right)^{D_{4}}\right)=2$ and $\operatorname{dim}\left(\operatorname{Harm}_{4}\left(\mathbb{R}^{n}\right)^{D_{n}}\right)=1$ for any $n \geq 5$. The following are $D_{n}$-invariant harmonic polynomials of degree 4 :

$$
f_{4}=\operatorname{sym}\left(x_{1}^{4}\right)-\frac{6}{n-1} \operatorname{sym}\left(x_{1}^{2} x_{2}^{2}\right) .
$$

The following is a $D_{4}$-invariant harmonic polynomial of degree 4 , which is linearly independent of $f_{4}$ :

$$
f_{4,2}=x_{1} x_{2} x_{3} x_{4}
$$

2. Degree 5 .

Note that $\operatorname{dim}\left(\operatorname{Harm}_{5}\left(\mathbb{R}^{5}\right)^{D_{5}}\right)=1$ and $\operatorname{dim}\left(\operatorname{Harm}_{5}\left(\mathbb{R}^{n}\right)^{D_{n}}\right)=0$ for any $n \neq 5$. The following is a $D_{5}$-invariant harmonic polynomial of degree 5 :

$$
f_{5}=x_{1} x_{2} x_{3} x_{4} x_{5} .
$$

3. Degree 6.

Note that $\operatorname{dim}\left(\operatorname{Harm}_{6}\left(\mathbb{R}^{6}\right)^{D_{6}}\right)=2$ and $\operatorname{dim}\left(\operatorname{Harm}_{6}\left(\mathbb{R}^{n}\right)^{D_{n}}\right)=1$ for any $n \neq 6$. The 
following is a $D_{n}$-invariant harmonic polynomial of degree 6 :

$$
f_{6}=\operatorname{sym}\left(x_{1}^{6}\right)-\frac{15}{n-1} \operatorname{sym}\left(x_{1}^{2} x_{2}^{4}\right)+\frac{180}{(n-1)(n-2)} \operatorname{sym}\left(x_{1}^{2} x_{2}^{2} x_{3}^{2}\right) .
$$

The following is a $D_{6}$-invariant harmonic polynomial of degree 6 , which is linearly independent of $f_{6}$ :

$$
f_{6,2}\left(v_{k}\right)=x_{1} x_{2} x_{3} x_{4} x_{5} x_{6} .
$$

\section{Substitute $v_{k}$ for $G$-invariant harmonic polynomials}

1. Degree 4.

For $1 \leq k \leq n-2$,

$$
f_{4}\left(v_{k}\right)=\frac{1}{k}\left(1-3 \frac{k-1}{n-1}\right)
$$

For $k=n-1, n$,

$$
f_{4}\left(v_{k}\right)=-\frac{2}{n}
$$

For $n=4$,

$$
f_{4,2}\left(v_{1}\right)=0, \quad f_{4,2}\left(v_{2}\right)=0, \quad f_{4,2}\left(v_{3}\right)=-\frac{1}{16}, \quad f_{4,2}\left(v_{4}\right)=\frac{1}{16} .
$$

2. Degree 5 .

For $n=5$,

$f_{5}\left(v_{1}\right)=0, \quad f_{5}\left(v_{2}\right)=0, \quad f_{5}\left(v_{3}\right)=0, \quad f_{5}\left(v_{4}\right)=-\frac{1}{25 \sqrt{5}}, \quad f_{5}\left(v_{1}\right)=\frac{1}{25 \sqrt{5}}$.

3. Degree 6.

For $1 \leq k \leq n-2$,

$$
f_{6}\left(v_{k}\right)=\frac{1}{k^{2}}\left(1-15 \frac{k-1}{n-1}+30 \frac{(k-1)(k-2)}{(n-1)(n-2)}\right) .
$$

For $k=n-1, n$,

$$
f_{6}\left(v_{k}\right)=\frac{16}{n^{2}}
$$

For $n=6$,

$$
\begin{aligned}
& f_{6,2}\left(v_{1}\right)=0, \quad f_{6,2}\left(v_{2}\right)=0, \quad f_{6,2}\left(v_{3}\right)=0, \quad f_{6,2}\left(v_{4}\right)=0, \\
& f_{6,2}\left(v_{5}\right)=-\frac{1}{216}, \quad f_{6,2}\left(v_{6}\right)=\frac{1}{216} \text {. }
\end{aligned}
$$

Theorem 4.10. There is no choice of $J, R$ and $w$ for which $\left(\mathcal{X}\left(D_{n}, J\right), w\right)$ is a Euclidean 8-design.

Proof. The following is a $D_{n}$-invariant harmonic polynomial of degree 8:

$$
f_{8}=\operatorname{sym}\left(x_{1}^{8}\right)-\frac{28}{n-1} \operatorname{sym}\left(x_{1}^{2} x_{2}^{6}\right)+\frac{70}{n-1} \operatorname{sym}\left(x_{1}^{4} x_{2}^{4}\right) .
$$

For $1 \leq k \leq n-2$,

$$
f_{8}\left(v_{k}\right)=\frac{1}{k^{3}}\left(1+7 \frac{k-1}{n-1}\right)
$$




\begin{tabular}{|c|c|c|c|c|c|}
\hline$|R|$ & $t$ & $n$ & $J$ & $r_{i}$ & $w_{i}$ \\
\hline 1 & 7 & 8 & $\{2,7\}$ & $r_{2}=r_{7}=1$ & $w_{2}=w_{7}=1$ \\
& 7 & 8 & $\{2,8\}$ & $r_{2}=r_{8}=1$ & $w_{2}=w_{8}=1$ \\
\hline 2 & 5 & 6 & $\{1,5\}$ & $r_{1}=1, r_{5} \neq 1$ & $w_{1}=1, w_{5}=\frac{9}{8 r_{2}^{4}}$ \\
& 5 & 6 & $\{1,6\}$ & $r_{1}=1, r_{6} \neq 1$ & $w_{1}=1, w_{6}=\frac{9}{8 r_{2}^{4}}$ \\
& 7 & 4 & $\{1,2,3,4\}$ & $r_{1}=r_{3}=r_{4}=1, r_{2} \neq 1$ & $w_{1}=w_{3}=w_{4}=1, w_{2}=\frac{1}{r_{2}^{6}}$ \\
\multicolumn{6}{c}{ TABLE 3. Tight Euclidean t-designs from $\mathcal{X}\left(D_{n}, J\right)$, where } \\
&
\end{tabular}

and for $k=n-1, n$,

$$
f_{8}\left(v_{k}\right)=\frac{8}{n^{3}} .
$$

Therefore $f_{8}\left(v_{k}\right)>0$ for all $k$.

Theorem 4.11. Assume $J$ contains $n$ or $n-1$. Then $\mathcal{X}\left(D_{n}, J\right)$ is not a tight Euclidean design except for the sets in Table 3.

Proof. By Theorem 4.10 and $D_{n}$-invariant harmonic polynomials, a proof is similar to that of Theorem 4.6.

Remark 4.12. The tight Euclidean designs in Tables $1,2,3$ are already known in [2, 4, 6, 7, 8, 10 .

Remark 4.13. For each $G=F_{4}, H_{3}, H_{4}, E_{6}, E_{7}, E_{8}$, by checking the cardinality of a union of several $v_{k}^{G}$, we can prove $\mathcal{X}(G, J)$ is not a tight Euclidean design except for known tight spherical designs [21, 23].

\section{Concluding Remarks}

In this paper we found some observations on invariant cubature formulas and Euclidean designs in connection with the Sobolev theorem. First, we gave an alternative proof of celebrated theorems by $\mathrm{Xu}$ on necessary and sufficient conditions for the existence of cubature formulas with radial symmetry. The new proof is much shorter and simpler compared to the original one by $\mathrm{Xu}$. Thus researchers in analysis will realize again the importance of the Sobolev theorem. Moreover our proof gives a general interpretation of the analytically-written conditions of $\mathrm{Xu}$ 's theorems, and so will promise researchers in algebra and combinatorics to be more familiar with Xu's theorems. Second we extended the Neumaier-Seidel theorem to invariant Euclidean designs, and thereby classified tight Euclidean designs obtained from unions of the orbits of the corner vectors. The classification generalizes Bajnok's theorem to other finite reflection groups beside groups of type $B$. Bajnok's theorem and results obtained in Section 4 may imply that invariant cubature formulas of high degree could hardly exist. Xu [29] pointed out, however, that the general Lie groups has been used for studying cubature formulas in a different setting - cubature rules on the fundamental domain of the group, which are for exponential or trigonometric functions - and they yield Gaussian type cubature for algebraic polynomials of very high orders; for instance see [16, 22] for details. We believe this direction of research in analysis will also motivate the study of cubature formulas in other areas of mathematics. 
Acknowledgements. The authors started writing this paper during their visit at the University of Texas at Brownsville, 2010, under the sponsorship of the Japan Society for the Promotion of Science. They would like to thank Oleg Musin for his hospitality. The authors would also like to thank Akihiro Munemasa, Eiichi Bannai for valuable comments to this work. The second author would like to express his sincerest appreciation to Yuan Xu for fruitful discussion about the content of Section $3([29)$.

\section{REFERENCES}

[1] B. Bajnok, On Euclidean designs. Adv. Geom. 6 (2006), 423-438.

[2] B. Bajnok. Orbits of the hyperoctahedral group as Euclidean designs. J. Algebraic Combin. 25 (2007), 375-397.

[3] Ei. Bannai. Private communication.

[4] Ei. Bannai, Et. Bannai. On Euclidean tight 4-designs. J. Math. Soc. Japan 58 (2006), 775804.

[5] Ei. Bannai, Et. Bannai. A survey on spherical designs and algebraic combinatorics on spheres. Europ. J. Combin. 30 (2009), 1392-1425.

[6] Ei. Bannai, Et. Bannai, M. Hirao, M. Sawa. Cubature formulas in numerical analysis and Euclidean tight designs. Europ. J. Combin. 31 (2010), 423-441.

[7] Et. Bannai. New examples of Euclidean tight 4-designs. Europ. J. Combin. 30 (2009), 655667.

[8] Et. Bannai. On antipodal Euclidean tight $(2 e+1)$-designs. J. Algebraic Combin. 24 (2006), 391-414.

[9] N. Bourbaki. Lie Groups and Lie Algebras: Chapters 4-6 (Elements of Mathematics). Springer, 2002).

[10] P. Delsarte, J.M. Goethals, J.J. Seidel. Spherical Codes and Designs. Geom. Dedicata 6 (1977), 363-388.

[11] P. Delsarte, J.J. Seidel. Fisher type inequalities for Euclidean t-designs. Lin. Algebra Appl. 114-115 (1989), 213-230.

[12] C.F. Dunkl, Y. Xu. Orthogonal Polynomials of Several Variables. Cambridge University Press, 2001.

[13] A. Erdělyi et al. Higher Transcendental Functions II. (Bateman Manuscript Project), MacGraw-Hill, 1953.

[14] J.M. Goethals, J.J. Seidel. Cubature formulae, polytopes, and spherical designs. The geometric vein, pp. 203-218, Springer, New York-Berlin, 1981.

[15] M. Hirao, M. Sawa. On minimal cubature formulae of small degree for spherically symmetric integrals. SIAM J. Numer. Anal. 47 (2009), 3195-3211.

[16] H. Li, Y. Xu. Discrete Fourier analysis on fundamental domain of $A_{d}$-lattice and on simplex in $d$-variables. J. Fourier Anal. Appl. 16 (2010), 383 - 433.

[17] H.M. Möller. Lower bounds for the number of nodes in cubature formulae, Numerische Integration (Tagung, Math. Forschungsinst., Oberwolfach, 1978). 221-230, Internat. Ser. Numer. Math. 45, Birkhäuser, Basel-Boston, Mass., 1979.

[18] I.P. Mysovskikh. Construction of cubature formulae (in Russian). Vopr. Vychisl. i Prikl. Mat. Tashkent 32 (1975), 85-98.

[19] I.P. Mysovskikh. Interpolatory Type Cubature formula (in Russian). Nauka, Moscow, 1981.

[20] A. Neumaier, J. J. Seidel. Discrete measures for spherical designs, eutactic stars and lattices. Nederl. Akad. Wetensch. Indag. Math. 50 (1988), 321-334.

[21] H. Nozaki. On the rigidity of spherical $t$-designs that are orbits of reflection groups $E_{8}$ and H4. Europ. J. Combin. 29 (2008), 1696-1703.

[22] J. Patera, R. Moody. Cubature formulae for orthogonal polynomials in terms of elements of finite order of compact simple Lie groups. arXiv:1005.2773

[23] A. Sali. On the rigidity of spherical $t$-designs that are orbits of finite reflection groups. Des. Codes Cryptogr. 4 (1994), 157-170.

[24] G.N. Salikhov. Cubature formulas for the hypersphere invariant under the 600-hedral group. Dokl. Akad. Nauk SSSR 223 (1975), 1075-1078. 
[25] S.L. Sobolev. Cubature formulas on the sphere which are invariant under transformations of finite rotation groups (in Russian). Dokl. Akad. Nauk SSSR 146 (1962), 310-313.

[26] A.H. Stroud. Approximate Calculation of Multiple Integrals. Prentice-Hall, Inc., Englewood Cliffs, N.J., 1971. xiii+431 pp.

[27] P. Verlinden, R. Cools. On cubature formulae of degree $4 k+1$ attaining Möller's lower bound for integrals with circular symmetry. Numer. Math. 61 (1992), 395-407.

[28] Y. Xu. Minimal cubature formulae for a family of radial weight functions. Adv. Comput. Math. 8 (1998), 367-380.

[29] Y, Xu. Private communication.

Graduate School of Information Sciences,, Tohoku University, Aoba-ku, Sendai 980 8579, JAPAN

E-mail address: nozaki@ims.is.tohoku.ac.jp

Graduate School of Information Sciences,, Nagoya University, Chikusa-ku, Nagoya 464-8601,, JAPAN

E-mail address: sawa@is.nagoya-u.ac.jp 